\title{
Predicting Reproductive Age with Biomarkers of Ovarian Reserve-How (and What) Are We Measuring?
}

\author{
Karl R. Hansen, MD, PhD ${ }^{1}$ \\ ${ }^{1}$ Section of Reproductive Endocrinology and Infertility, Department of \\ Obstetrics and Gynecology, University of Oklahoma Health Sciences \\ Center, Oklahoma City, Oklahoma \\ Semin Reprod Med 2013;31:416-426
}

\begin{abstract}
Address for correspondence Karl R. Hansen, MD, PhD, Section of Reproductive Endocrinology and Infertility, Department of Obstetrics and Gynecology, University of Oklahoma Health Sciences Center, P.O. Box 26901, WP 2410, Oklahoma City, OK 73126

(e-mail: karl-hansen@ouhsc.edu).
\end{abstract}

\begin{abstract}
Keywords

- ovarian reserve

- nongrowing follicles

- primordial follicles

- anti-Müllerian hormone

- antral follicle count

Predicting the reproductive lifespan of an individual woman remains an elusive, yet clinically important, goal. The development of models and staging systems that accurately determine the end of natural fertility and the anticipated age of menopause will represent a significant advance in our ability to counsel women regarding family planning issues and in the individualization of risk assessment. Recent histological and longitudinal investigations have demonstrated a significant relationship between commonly used clinical markers of ovarian reserve and the true ovarian reserve, as assessed by the ovarian nongrowing follicle count and the age of menopause, respectively. Models and staging systems that have been developed based on these findings represent important advances in the field of women's health and promise to provide additional insights into the process of reproductive aging in general. Although the models developed to date appear to improve the prediction of the age of menopause as compared with chronological age alone, wide confidence intervals in the predicted age of menopause and less accurate predictions at ages remote from menopause limit their clinical utility for the individual woman. Future longitudinal and histological investigations are necessary to improve the accuracy of models of reproductive aging.
\end{abstract}

Reproductive aging in women refers to the progressive loss of fertility and ovarian endocrine function which ultimately results in the menopause. Although the process is certainly not new, events over the last few decades have brought renewed interest in developing a better understanding of the reproductive aging process and its clinical prediction. First, socioeconomic and demographic trends have resulted in many women delaying childbearing until their late 30s and early 40s, when natural fertility is compromised relative to younger reproductive-aged women. ${ }^{1-3}$ Educational campaigns have increased the awareness of the general public to this phenomenon and have fueled a desire to better predict the end of natural fertility. ${ }^{4}$ Second, a broader understanding has emerged regarding the relationship between an early age of menopause and an increased risk for medical problems including colorectal cancer, osteoporosis, and cardiovascular and urogenital disease. ${ }^{5-8}$ Conversely, a delayed age of menopause may be associated with an increased risk of cancers of the breast, endometrium, and ovary. ${ }^{9,10}$ Finally, large numbers of women are passing through the menopausal transition as the "baby boom" population ages, with the attendant symptoms that may significantly impact quality of life. In sheer population numbers, the prediction of the reproductive lifespan in now compelling-a relatively modern occurrence given that only in the last approximately 100 years has the average woman lived to age 50 and actually experienced the menopause. ${ }^{11}$ Thus, understanding and predicting the reproductive aging process is much more than an academic exercise-it has real-world applications and implications.
Copyright (C) 2013 by Thieme Medical Publishers, Inc., 333 Seventh Avenue, New York, NY 10001, USA. Tel: +1(212) 584-4662.
DOI http://dx.doi.org/ $10.1055 / \mathrm{s}-0033-1356477$. ISSN 1526-8004. 
The average age of menopause is 51 years; however, there is a wide variation between individuals, with some women entering menopause by the age of 40 and others having menstrual cycles into their mid to late $50 \mathrm{~s}^{12}$ Unfortunately, the progressive loss of ovarian function and fertility is largely a silent process. Changes in menstrual cycle characteristics such as shortening of the menstrual cycle length are subtle initially. ${ }^{13}$ By the time a women experiences oligomenorrhea associated with elevated gonadotropins, she is near the end of the reproductive aging process and her fertility is severely compromised. ${ }^{14}$ In reality though, her fertility was compromised many years earlier. With the understanding that chronological age is a crude predictor of the reproductive aging process, considerable interest has been directed toward identifying biomarkers as well as developing models and staging systems that might better characterize and predict the reproductive lifespan. Such models would have many potential applications. It has been hypothesized that the interval of time between the end of natural fertility and menopause is fixed, regardless of the exact age at which menopause occurs (-Fig. 1). ${ }^{15}$ Therefore, an accurate model that forecasts the age of menopause may also predict the end of natural fertility. Beyond family planning, such a model may also be useful for women with a history of prior exposure to chemotherapy, radiation, or ovarian surgery. This article reviews our current understanding of the reproductive aging process and the utility of common clinical tests of ovarian reserve in assessing reproductive age.

\section{The Biology of Reproductive Aging: What Are We Attempting to Measure?}

Although neuroendocrine changes have been described late in the reproductive aging process, there is broad consensus that the primary mechanism behind reproductive aging is the

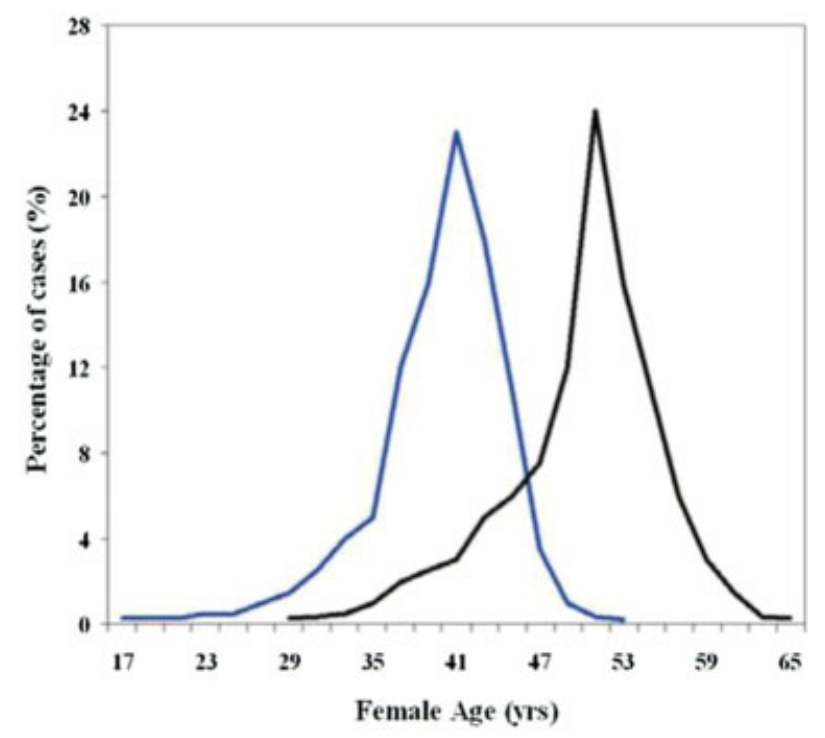

Figure 1 Distribution curves for observed age at last child birth (proxy variable for natural sterility, left line) and age at menopause (right line). Reprinted with permission from Lambalk et al. ${ }^{15}$ progressive loss of microscopic ovarian follicles. ${ }^{1,13,16}$ Therefore, modeling or predicting the reproductive lifespan is, in essence, an attempt to predict the number of remaining ovarian follicles in a given woman and the point at which this follicular reserve will be exhausted. The ovarian follicle is the functional/anatomical unit of the ovary, consisting of a primary oocyte surrounded by granulosa cells. The ovarian primordial follicle (PF) consists of a primary oocyte surrounded by a single layer of flattened granulosa cells. Intermediate follicles consist of a primary oocyte surrounded by some flattened and some cuboidal granulosa cells, and primary follicles consist of a primary oocyte surrounded by an entirely cuboidal layer of granulosa cells. ${ }^{17}$ Collectively, this group of the three follicle types is referred to as the pool of resting or nongrowing follicles (NGFs). Some investigators consider the PF pool to represent the ovarian reserve, whereas others believe the reserve includes all NGFs. ${ }^{15,18}$ Differences of opinion notwithstanding, it is clear that the true ovarian reserve is represented by one or both of these groups, with investigations utilizing PF counts or total NGF counts as the outcome of interest reaching similar conclusions.

Follicles are selected or recruited from this pool of NGFs, progressively moving through the preantral, early antral, antral, and ultimately the Graafian follicle stage. The earliest stages of this process are gonadotropin independent, with the vast majority of follicles undergoing atresia rather than ovulation. ${ }^{13}$ Beyond the quantitative loss of NGFs associated with reproductive aging, a considerable loss in quality of the remaining oocytes also occurs during this process, as demonstrated by an increased rate of aneuploid oocytes and derived embryos in the setting of the assisted reproductive technologies (ART). ${ }^{19,20}$ Given that the reproductive lifespan is dictated by the initial size of the ovarian follicle pool in a given woman and its rate of loss, considerable efforts have been put forth to develop a better understanding of this process.

\section{Modeling the Depletion of NGFs: Histological Investigations}

Direct measures of ovarian PFs and total NGFs in women are relatively rare due to the challenges associated with the execution of these types of investigations. Appropriate tissue for histological examination is exceedingly difficult to obtain, and the processing of ovarian tissue and the counting of ovarian follicles is demanding and time-intensive. Older model-based techniques involved embedding the ovaries in paraffin followed by serial sectioning and counting between 1 in 10 and 1 in 200 sections. ${ }^{21-23}$ The number of follicles counted was then multiplied by the inverse of the sampling fraction to obtain a raw estimate of the total NGF number within a given ovary. Raw counts were then frequently multiplied by a correction factor to obtain a "corrected" follicle count. ${ }^{21-23}$ The application of these correction factors (applied to counts of some, but not all ovaries in many investigations) attempts to correct for the observation that small particles (follicles) tend to be underrepresented in tissue sections and undercounted, whereas larger particles (follicles at more advanced stages) tend to be over counted 
due to their appearance in multiple thin tissue sections. Whether the incorporation of these correction factors into estimates of ovarian follicle counts improves their accuracy cannot be ascertained, as the model cannot be tested by the model itself. As a result of these challenges, to our knowledge, only three investigations have ever estimated NGF counts for more than forty ovaries..$^{21,22,24}$ Only one of these investigations has utilized modern stereology techniques which do not require the incorporation of correction factors. ${ }^{24}$ These modern stereology methods, combining the fractionator and optical disector tools, have become the standard in structural analysis. ${ }^{25,26}$

Attempts to model ovarian NGF decay have frequently incorporated follicle counts from multiple studies. Limitations of this approach include the systematic bias introduced with the variable use of correction factors as described above, the lack of appropriate interobserver validation studies, and the combination of NGF count estimates from investigations that used similar, but not identical counting techniques. Earlier investigations using these methodologies suggested that the decline in the log-transformed ovarian NGF count exhibited a biphasic exponential decline, with approximately 1 to 2 million follicles at birth and a break point in the mid-30s at approximately 25,000 NGFs when the decay curve suddenly developed a more negative slope. ${ }^{23} \mathrm{~A}$ more recent investigation which also combined NGF counts from multiple studies suggested that the change in NGF counts from conception to menopause was best characterized by a fiveparameter asymmetric double Gaussian cumulative curve. ${ }^{27}$ As described above, models derived from the combined data from multiple studies must be interpreted with caution. In essence, they are the histological equivalent of determining the U.S. census using different methods in different regions and combining the results. In spite of the questionable validity of this approach, it remains common due to the scarcity of histological investigations.

In contrast to models derived from combined data, the largest study $(n=122)$ from a single group of investigators utilizing modern morphometric techniques has suggested that the decline in the log-transformed ovarian NGF count from birth to menopause is best described by a simple power function (-Fig. 2$)^{24}$ :

$\log ($ NGF count $)=(-0.00019) \times(\text { age in years })^{2.452}+5.717$

The power model is a robust fit to the observed data $\left(R^{2}=0.84\right)$, and suggests that there is no sudden change in the rate of ovarian NGF loss associated with aging, but rather a smooth change. Additionally, the model predicts, on average, approximately 520,000 NGFs present in both ovaries at birth and approximately 750 follicles remaining at the time of menopause. ${ }^{24}$ An independent dataset developed with modern stereology techniques is necessary to validate the power model of ovarian NGF decline associated with aging. Although log-transformation of ovarian NGF counts is necessary from a modeling standpoint, this approach can easily obscure the tremendous loss of NGFs associated with aging; a loss readily appreciated when reviewing the non-log transformed data (-Fig. 3). By the age of 33 on average, approximately $90 \%$ of the ovarian NGFs are depleted. ${ }^{24}$

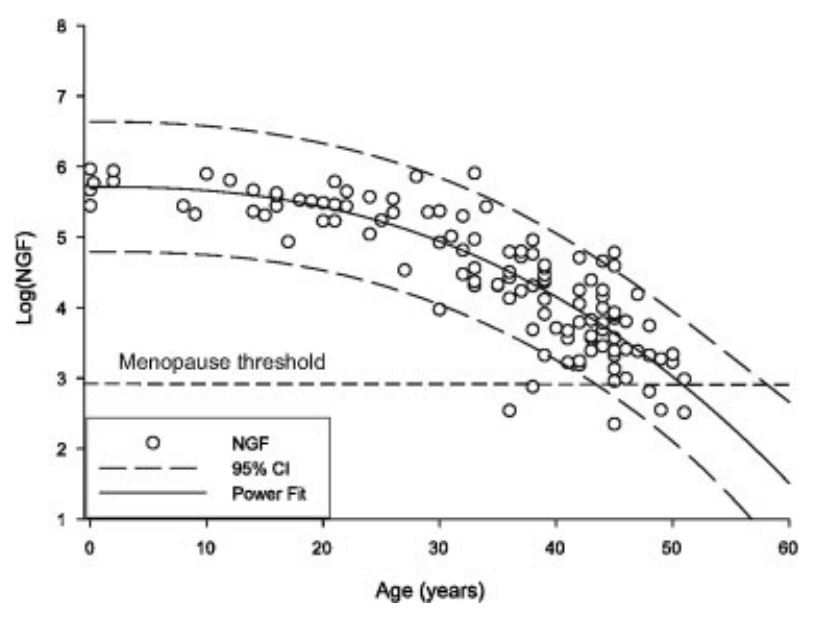

Figure 2 Power-model of ovarian nongrowing follicle (NGF) decay. The log of the ovarian NGF number is plotted versus age (years). The solid line indicates the fitted model with dashed lines representing the $95 \%$ confidence interval $(n=122)$. Reprinted with permission from Hansen et al. ${ }^{24}$

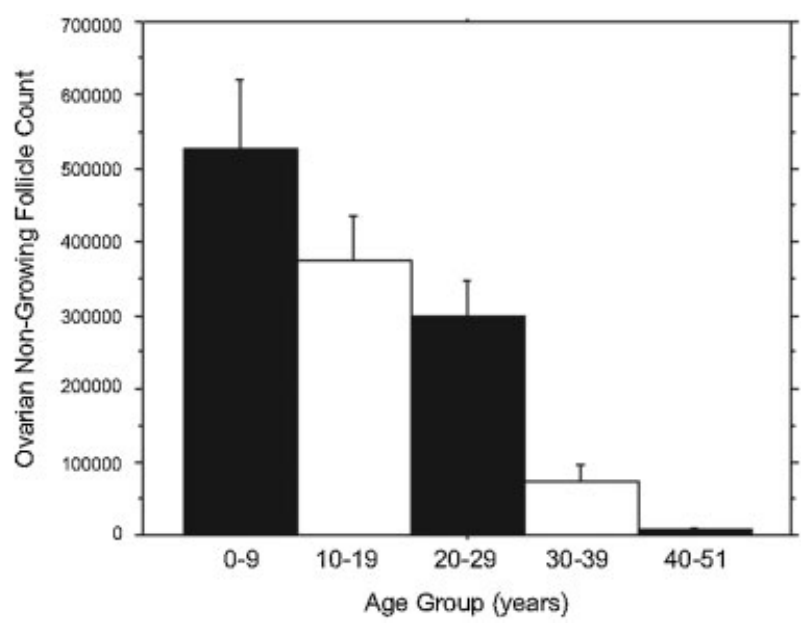

Figure 3 Histogram representation of the average nongrowing follicle (NGF) count for subjects in different age groups $(n=122)$. The height of the bar represents the average NGF count for the given age group \pm SEM. Adapted with permission from Hansen et al. ${ }^{24}$

\section{“Ovarian Reserve" Tests-What are We Measuring?}

The concept of "ovarian reserve" testing is deeply rooted in the ARTs. With the understanding that chronological age is a relatively poor predictor of how an individual woman might respond to gonadotropin stimulation in the setting of in vitro fertilization (IVF), multiple tests have been developed to predict the ovarian response. Although the ideal outcome of interest would be live-births in these investigations, most have used surrogate markers, including the number of oocytes retrieved or the peak estradiol level following gonadotropin stimulation. Commonly used clinical markers of ovarian reserve include basal serum levels of follicle-stimulating hormone (FSH), basal estradiol, basal inhibin $\mathrm{B}$, the 
ovarian antral follicle count (AFC) as determined by transvaginal ultrasound examination, and serum levels of antiMüllerian hormone $(\mathrm{AMH}){ }^{28-41}$ Other less frequently utilized tests include the clomiphene citrate challenge test and the gonadotropin-releasing hormone $(\mathrm{GnRH})$ agonist challenge test. $^{42-45}$

Although all of these tests have some predictive value with regards to the outcome of gonadotropin stimulation from a quantitative standpoint (e.g., number of oocytes obtained following treatment), they appear to have much less power at predicting pregnancy and live-births. ${ }^{46}$ Most investigations have suggested that serum levels of AMH and the ovarian AFC have the most predictive power from a quantitative standpoint, and a recent meta-analysis has suggested there is no benefit to obtaining multiple markers of ovarian reserve rather than a single marker to predict responsiveness. ${ }^{46,47}$ Few investigations have incorporated chronological age as a variable to improve the predictive value of a given biomarker, although the construction of efficiency curves, which include the impact of chronological age and ovarian reserve markers, may be useful in the identification of threshold levels of a given marker beyond which pregnancy is unlikely. ${ }^{48,49}$

While these clinical tests are commonly referred to as "ovarian reserve" tests, they are more accurately measures of ovarian responsiveness. In other words, their utility is to predict response in the artificial setting of IVF. Conversely, the ovarian reserve, in its truest sense, is referring to the size of the remaining ovarian NGF pool. This distinction between ovarian responsiveness and the true ovarian reserve is critical. Both are clinically relevant and are definitely related. However, the pool of recruitable or selectable follicles (i.e., the ovarian responsiveness) at any given point in time may be influenced by factors other than the size of the ovarian NGF pool, such as the dose of gonadotropins utilized in ART, the specific stimulation protocol, and likely many other unknown factors. This distinction may help explain why commonly used clinical markers of "ovarian reserve" may outperform age in the clinical prediction of ovarian responsiveness in the setting of IVF whereas chronological age is the best single predictor of the true ovarian reserve (i.e., the ovarian NGF pool). 29,37,39,50

With the understanding of the distinction between ovarian responsiveness and the true ovarian reserve, what is the evidence that these clinical biomarkers are actually reflective of the ovarian NGF pool, and thus, the reproductive lifespan? Multiple lines of evidence have supported a correlation between ovarian reserve markers and the true ovarian reserve. However, only recently have histological and longitudinal investigations confirmed this relationship.

\section{Examining the Circumstantial Evidence}

\section{Follicle-Stimulating Hormone}

FSH is a dimeric glycoprotein produced by the anterior pituitary known to regulate the recruitment and growth of ovarian follicles from the antral stage to the Graafian follicle. FSH has been studied extensively as a marker of ovarian responsiveness, with some investigations suggesting it outperforms chronological age in the prediction of ART outcome from a quantitative standpoint in univariate analyses. ${ }^{29,37,39}$ Multiple cross-sectional investigations have demonstrated an increase in serum levels of FSH associated with increasing chronological age. ${ }^{51,52}$ Although these increases can be demonstrated across the menstrual cycle, they are most prominent in the early follicular phase. ${ }^{53}$ Longitudinal investigations have confirmed these findings, with variable increases in FSH being associated with subtle shortening of the follicular phase of the menstrual cycle, and more dramatic increases associated with menstrual cycle changes characteristic of the late perimenopause (Stages of Reproductive Aging Workshop [STRAW] stage-1) (- Fig. 4). ${ }^{54-59}$ Gradual increases in FSH are observed within 10 years of menopause. ${ }^{59}$ These observations strongly suggest that serum levels of FSH are correlated with reproductive age, but the relatively late onset of these changes relative to the reproductive aging process and the cycle-specific nature of its measurement highlight its limitations as marker of true ovarian reserve.

\section{Inhibin B}

Inhibin $B$ is a dimeric polypeptide produced by the granulosa and theca cells of the developing cohort of antral follicles, whereas inhibin A is primarily a product of the developing dominant follicle and the corpus luteum. ${ }^{60}$ Both are known to exert a negative feedback at the level of the hypothalamus and pituitary, ultimately resulting in the decrease in FSH secretion. ${ }^{61,62}$ Like FSH, the inhibins have been evaluated extensively as a marker of ovarian responsiveness, although they have never enjoyed the popularity of FSH due to a variety of reasons. Early assays for the inhibins were not as specific for inhibin A versus B, and levels of inhibin B associated with decreased responsiveness in the setting of ART were near the limits of detection of the early assays. ${ }^{63,64}$ Additionally, some investigations suggested its predictive value was no greater than that of FSH. ${ }^{46,64,65}$ Inhibin B levels are also cycle phasedependent, with the highest levels observed in the early follicular phase and mid-cycle. Cross sectional investigations have demonstrated lower early follicular phase levels of inhibin B in older as opposed to younger reproductive-aged women. ${ }^{53}$ These findings have been confirmed in longitudinal investigations. ${ }^{59,66}$ Inhibin B concentrations fall below the limits of detection within 4 to 5 years of the menopause, and significant decreases are variably detected by the late reproductive years (STRAW stages-3a). ${ }^{55-59}$ Although these findings would suggest that inhibin B levels may reflect the size of the remaining pool of NGFs, some investigations have suggested the decline in its level is not gradual, which would limit its value as a biomarker of true ovarian reserve. ${ }^{67}$

\section{The Ovarian Antral Follicle Count}

The ovarian AFC as determined by transvaginal ultrasound examination has been widely evaluated as a marker of ovarian responsiveness. ${ }^{34-38}$ The AFC is the number of antral follicles between 2 and $10 \mathrm{~mm}$ in size within both ovaries observed on transvaginal ultrasound examination, and is reflective of the pool of potentially recruitable follicles following 


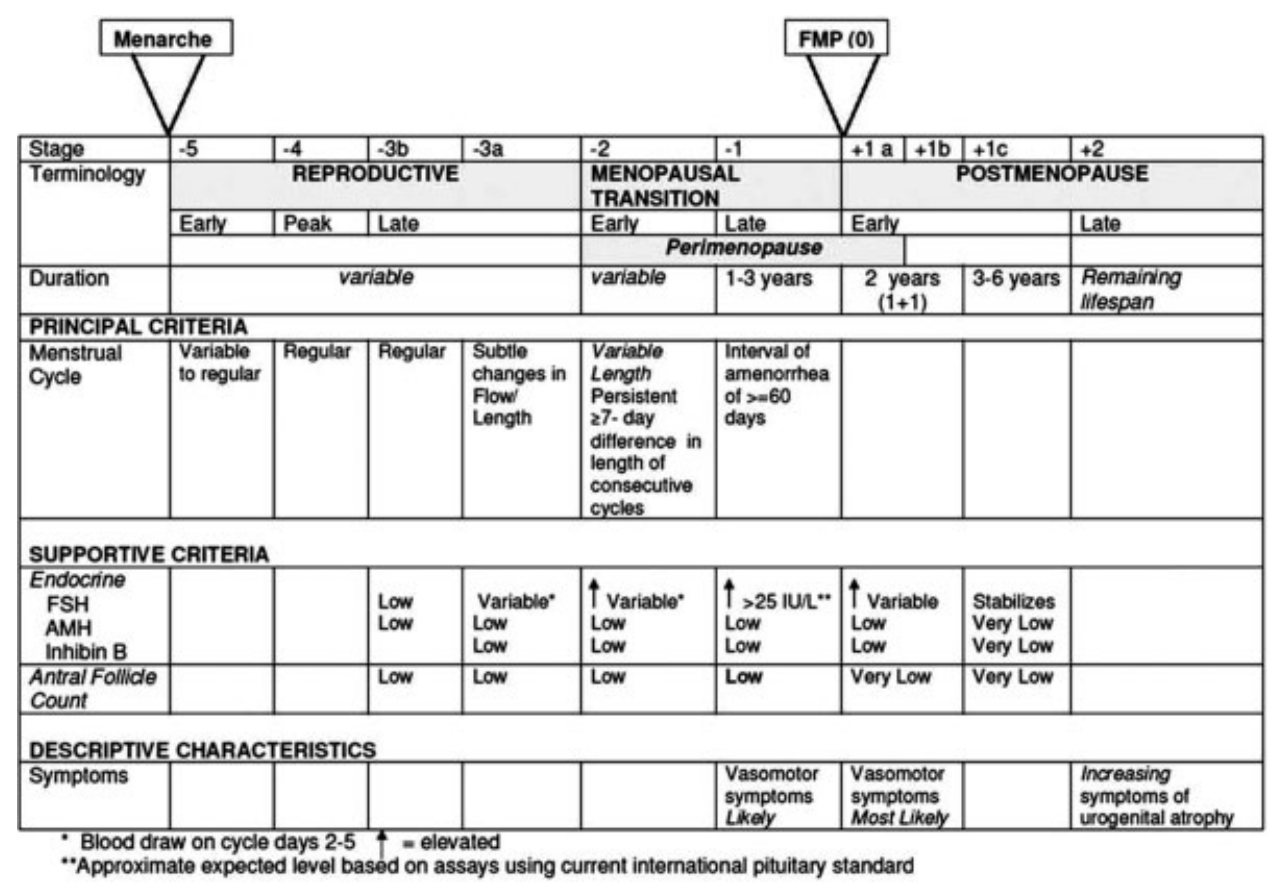

Figure 4 The Stages of Reproductive Aging Workshop + 10 staging system for reproductive aging in women. Reprinted with permission from Harlow et al. ${ }^{55-58}$

gonadotropin stimulation. The AFC is highly predictive of the response of an individual patient to gonadotropins in the setting of IVF, and along with serum levels of AMH, is one of the best single predictors of stimulation outcome from a quantitative perspective. ${ }^{37,39,46}$ Multiple cross-sectional investigations have demonstrated a decrease in the ovarian AFC associated with increasing age. ${ }^{68,69}$ In addition to the agerelated decrease in the ovarian AFC, the AFC is also noted to decrease with progressive changes in menstrual cycle characteristics associated with the menopausal transition. ${ }^{70,71}$ The gradual decline in the ovarian AFC associated with aging and its cycle-phase independence makes it a more attractive marker of reproductive aging as compared with serum levels of FSH and inhibin $\mathrm{B}^{69}$

\section{Anti-Müllerian Hormone}

AMH is produced by the granulosa cells of preantral and early antral follicles, and serum levels fluctuate minimally throughout the menstrual cycle. ${ }^{72,73}$ Because it is secreted by follicles at the transition point between resting and growing follicles, it is one of the best single markers of ovarian responsiveness. Many reports in the literature indicate serum levels of AMH outperform all other ovarian reserve tests in the prediction of ART outcome with the possible exception of the AFC, the two of which are highly correlated. ${ }^{39,41,46,74}$ Multiple cross-sectional and longitudinal investigations have demonstrated a gradual decline in serum AMH level associated with increasing chronological age. ${ }^{39,59,75-77}$ As with the ovarian AFC, AMH is a prime candidate as a marker for reproductive age due to its gradual decline with advancing age, although some investigations have suggested this gradual decline does not begin until the age of approximately 25 to 30 years. $^{78,79}$
Additionally, these decreases have been noted in longitudinal investigations wherein significant changes were not observed in other ovarian reserve markers. ${ }^{80}$ These observations suggest that AMH may be a useful marker in younger (at least at age 30 and older) reproductive-aged women. Progressive decreases in serum levels of AMH are also noted with advancing stage of the STRAW staging system, with levels below the limits of detection reached within 5 years of menopause. ${ }^{59,71,75}$ Although AMH appears promising as a measure of the reproductive lifespan, the lack of standardized assays and sufficient assay sensitivity remain problematic. Furthermore, since serum AMH levels appear to be reflective of the early growing follicular pool, pathological states associated with abnormal follicular development (e.g., hypogonadotropic hypogonadism) may result in conditions in which the relationship between serum AMH and the NGF pool may be altered. $^{81}$

\section{The Association between Early Menopause and Poor Stimulation in the Setting of IVF}

Since commonly used clinical test of ovarian reserve are highly predictive of ovarian responsiveness in the setting of ART, an earlier menopause in women with a poor response to gonadotropins would serve as indirect evidence of an association between ovarian reserve tests results and the reproductive lifespan. Several case control and retrospective cohort studies have supported this hypothesis. ${ }^{82-84}$ The largest of these investigations, which included 4601 subjects with a median follow-up of 5.5 years, suggested that the adjusted odds ratio for having entered the menopausal transition or natural menopause for women with a poor response to stimulation as compared with normal responders was 3.1 
(95\% confidence interval $[\mathrm{CI}] 2.4-3.8) .{ }^{82}$ A second smaller investigation with similar follow-up times by Lawson et al reached similar conclusions. ${ }^{83}$ Although these studies suggest ovarian reserve markers are related to the reproductive lifespan, this evidence must be considered circumstantial and preliminary.

\section{Examining the Evidence: Histological Investigations}

Investigations into the relationship between commonly used clinical markers of ovarian reserve and the actual ovarian PF or NGF pool are exceedingly rare. This scarcity is partially explained by the reasons outlined above for histological studies in general. However, an additional challenge faced in these investigations is obtaining ovarian reserve markers for study participants prior to histological examination of the ovary. In other words, tissue obtained from autopsy or organ donation, while appropriate for some investigations, is unsuitable to address the question at hand. Only women undergoing elective oophorectomy are appropriate participants, and ovarian reserve assessments must be performed shortly prior to surgery.

Only two prior studies have directly addressed the relationship between ovarian reserve markers and ovarian PF count in women. The first investigation did not identify a significant relationship between the GnRH agonist stimulation test, the clomiphene citrate challenge test or basal FSH levels and the number of ovarian follicles per cubic centimeter. ${ }^{85}$ Limitations of this investigation included its small sample size $(n=22)$ and the use of older counting techniques. Furthermore, because the ovarian AFC and serum levels of $\mathrm{AMH}$ were not in use at the time as ovarian reserve tests, the two best markers were not evaluated.

The only other investigation of the relationship between ovarian reserve markers and the ovarian PF and NGF counts demonstrated a significant correlation between follicle counts and serum levels of $\mathrm{AMH}$, inhibin $\mathrm{B}, \mathrm{FSH}$ and the ovarian AFC (-Table 1). ${ }^{50}$ Partial correlations controlling for chronological age, the marker most strongly correlated with the ovarian PF count, demonstrated that the ovarian AFC and serum levels of AMH add predictive power in the assessment of the ovarian PF count beyond the contributions of age alone
(-Table 1). Although the AFC slightly outperformed AMH in this investigation, the difference between the two markers was small. ${ }^{50}$

Characteristic menstrual cycle changes associated with the menopausal transition process can also be considered biomarkers of ovarian reserve. Cross-sectional and longitudinal investigations have characterized these changes. ${ }^{86-90}$ Most commonly, a woman initially experiences a shortening in menstrual cycle length with advancing age due to a shortening of the follicular phase. ${ }^{86}$ Carefully designed studies have demonstrated that this shortening of the follicular phase is due to the advanced selection of the dominant follicle in older as compared with younger reproductive-aged women. ${ }^{91}$ In the latter transition, intervals of oligomenorrhea and amenorrhea occur due to anovulation. The STRAW staging system is the most widely utilized staging system of the reproductive aging process, adding significantly to the characterization of this process. ${ }^{55-58,92}$ Recent histological investigations have validated STRAW stage 4 through stage 1 as defined by these characteristic menstrual cycle changes, with significant decreases in the ovarian PF count noted with advancing STRAW stage (-Fig. 5) ${ }^{71}$ However, as with biomarkers of ovarian reserve, there is considerable overlap in the ovarian PF count between the stages, and the PF count alone is inadequate to assign a given individual to a single stage.

While histological investigations have provided important insights into the process of reproductive aging and the relationship between biomarkers of ovarian reserve and the true ovarian reserve, it is important to have a full understanding of their strengths and limitations. Histological investigations measure the actual outcome of interest, the ovarian PF and NGF pool, rather than relying on surrogate markers of reproductive age. Although the menopause is certainly an outcome of interest, the lack of vaginal bleeding in a late reproductive-aged woman may be due to factors other than ovarian failure, such as hyperprolactinemia or thyroid disorders. Conversely, vaginal bleeding may occur in the absence of significant ovarian follicular activity due to pathology, or due to the peripheral conversion of androgens to estrogens, a problem more prevalent in our increasingly obese society. Limitations include the cross-sectional nature of all histological studies. Additionally, participants in these investigations may not be considered "normal," as they all have some

Table 1 Univariate and partial correlations of endocrine parameters, ultrasound determined AFC and log 10 primordial follicle count $(n=42)$

\begin{tabular}{|l|l|l|l|l|l|l|l|l|}
\hline \multicolumn{3}{|c|}{ Step 1 } & \multicolumn{3}{c|}{ Step 2: Adjusting for age } & \multicolumn{3}{c|}{ Step 3: Adjusting for age + AFC } \\
\hline & $R$ & $p$-Value & & $R$ & $p$-Value & & $R$ & $p$-Value \\
\hline Age & -0.80 & $<0.0001$ & AMH & 0.48 & 0.0017 & AMH & 0.26 & 0.1158 \\
\hline AMH & 0.72 & $<0.0001$ & Inhibin B & 0.23 & 0.1459 & Inhibin B & 0.09 & 0.5756 \\
\hline Inhibin B & 0.40 & 0.0100 & FSH & -0.15 & 0.3674 & FSH & -0.04 & 0.8145 \\
\hline FSH & -0.32 & 0.0402 & Estradiol & 0.18 & 0.2555 & Estradiol & 0.26 & 0.1064 \\
\hline Estradiol & 0.12 & 0.4575 & AFC & 0.53 & 0.0005 & & & \\
\hline AFC & 0.78 & $<0.0001$ & & & & & & \\
\hline
\end{tabular}

Abbreviations: AFC, antral follicle count; $\mathrm{AMH}$, anti-Müllerian hormone; FSH, follicle-stimulating hormone. Reprinted with permission from Hansen et al. ${ }^{50}$ 

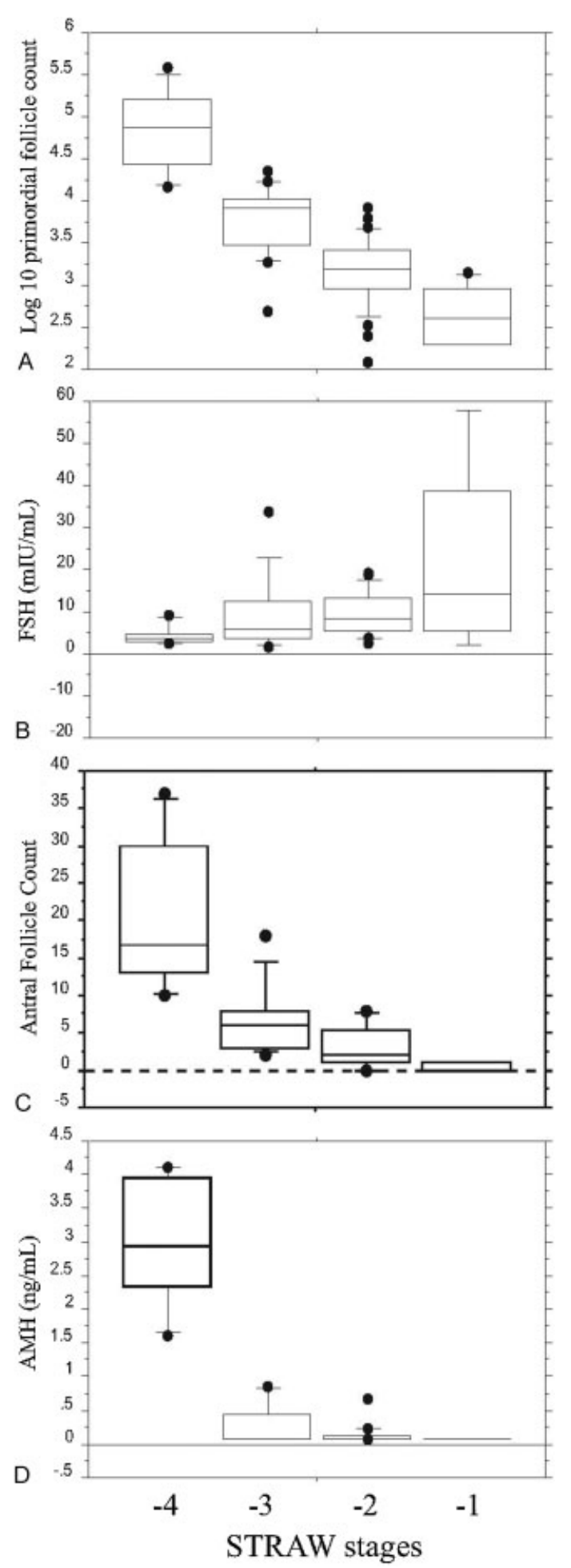

Figure 5 Box plots of log10-transformed ovarian primordial follicle counts, total ovarian antral follicle counts, and biomarkers of ovarian reserve for STRAW stage 4 through stage 1. (A) Log 10 primordial follicle count: $p<0.0001$ between all stages except $-2 \mid-1$, where $p=0.0074$; (B) FSH: $p=0.0036$ between stages $-4 /-1$, all others are not significantly different; $(C)$ antral follicle count: $p<0.0001$ between all stages except $-3 /-2,-3 /-1$, and $-2 /-1$ which are not significantly different; (D) AMH: $p<0.0001$ between all stages except $-3 /-2,-3 /-1$, and $-2 /-1$ which are not significantly different. Adapted with permission from Hansen et al. ${ }^{71} \mathrm{AMH}$, anti-Müllerian hormone; FSH, follicle-stimulating hormone; STRAW, Stages of Reproductive Aging Workshop. indication for a surgical procedure. Therefore, it is uncertain if the findings can be more broadly applied to women without such indications.

Finally, it is important to note that the histological assessment of ovarian reserve is a research tool only. Because of the irregular distribution of ovarian primordial and NGFs within the ovarian cortex, ovarian biopsies are not an adequate technique for the assessment of ovarian reserve, largely intact ovaries are required. ${ }^{93}$ To state the obvious-it is not practical to remove the ovary(ies) of a women to determine the status of her ovarian reserve. Ultimately the development of clinically useful tools and models depends upon the longitudinal investigation of readily available clinical markers utilizing the outcome of the final menstrual period.

\section{Examining the Evidence: Longitudinal Investigations}

Several recent investigations with longitudinal follow-up have evaluated the relationship between clinical markers of ovarian reserve and the occurrence of menopause. Broekmans et al initially demonstrated a significant agreement between the predicted and observed age at menopause in a model developed using the ovarian AFC in a cohort of 163 regularly cycling women. ${ }^{94}$ However, the predictive value of the model was limited except in the setting of low AFC for chronological age. Additionally, follow-up studies by the same group of investigators have demonstrated that the AFC was only predictive of the age of menopause in a univariate analysis; with chronological age and AMH the only significant predictors in a multivariate analysis. ${ }^{95,96}$ Sowers et al, following a cohort of 50 women through the menopause, demonstrated that baseline AMH levels were highly associated with the age of menopause, with serum levels falling below the limits of detection within 5 years of the final menstrual period. ${ }^{59}$ Although inhibin B levels were also low or undetectable within 5 years of menopause, they were less predictive of the final menstrual period than was AMH. ${ }^{59}$

More recent and larger investigations have confirmed the relationship between serum AMH concentrations and the reproductive life-span. Tehrani et al followed a group of 266 fertile women aged between 20 and 50 for an average of 6 years. ${ }^{97}$ In this time frame, 63 women experienced their final menstrual period. This investigation demonstrated a reasonable agreement between the observed and predicted age of menopause based on a single AMH measurement, with lower accuracy of the prediction noted at the extremes of age of anticipated menopause. An investigation of a larger cohort of fertile women (1,015 women) with 277 occurrences of menopause by the same group confirmed these findings utilizing a model that included both age and serum AMH as predictors of the final menstrual period. ${ }^{98} \mathrm{AMH}$ and chronological age were also significant predictors of the age of menopause in a recent investigation of 401 women followed through the menopausal transition in the Penn Ovarian Aging Study. ${ }^{99}$ A more recent study by the same group of investigators has suggested that the rate of change of $A M H$, in addition to chronological age, may improve the precision of 
estimates of the final menstrual period as compared with models utilizing a single AMH measurement. ${ }^{100}$ Similar to the Tehrani et al investigations, ${ }^{97}$ less precision in the estimate of the final menstrual period was observed at more remote intervals from the menopause.

Although these clinical investigations have provided powerful evidence of the relationship between ovarian reserve biomarkers (particularly AMH) and the true ovarian reserve, limitations of their clinical applicability should be noted. Currently there is no international standard for $\mathrm{AMH}$, and the commercially available assays lack the adequate sensitivity to be useful near the end of the reproductive lifespan. ${ }^{55-58}$ As discussed by the authors of many of these studies, the clinical prediction of the age of menopause tends to be more accurate at times closer to the menopause, and in some cases, at more advanced chronological age as compared with younger reproductive-aged women. For example, at a similar AMH level for a given woman $(<0.20 \mathrm{ng} / \mathrm{mL})$, the $95 \% \mathrm{CI}$ for the time to menopause is 4.20 to 6.33 years (median 5.99 years) if she is 45 to 48 years old, whereas it is 3.31 to 12.73 years (median 9.94 years) if she is 35 to 39 years old. ${ }^{99}$ Beyond the wide confidence intervals associated with some model-based predictions, it is important to note that even at the same AMH level, the time, on average, until the final menstrual period is significantly influenced by chronological age itself. In essence, the addition of AMH measurements to the derived models adds to the predictive power of age in the assessment of the reproductive lifespan, but it does not replace age. These observations are entirely consistent with the power model of ovarian NGF depletion, wherein $84 \%$ of the decline in NGF counts associated with increasing age can be explained by age alone. ${ }^{24}$ Strikingly, Tehrani et al $^{97}$ reached similar conclusions, with age alone having an adequacy of $84 \%$ to predict age at menopause correctly, increasing to $92 \%$ when $\mathrm{AMH}$ was added to their model. ${ }^{98}$ Although histological investigations have demonstrated that clinical ovarian reserve tests add to the predictive power of chronological age in partial correlation analyses, whether or not they can improve the predictive value of the power model of NGF decay awaits further histological studies. ${ }^{50}$

In addition to the above considerations, it is also important to note the population of women included in these longitudinal investigations. Although "normal" women may be an ideal population in which to study the reproductive aging process, it is unclear if the resulting models can be generalized to those commonly seen in clinical practice (e.g., infertility patients). Finally, it is also important to have an understanding of when these models may be more likely to give less accurate predictions. As described above, AMH levels may only experience a relatively predictable decline beginning at approximately 25 to 30 years of age. Therefore, applying the above models to women in younger age groups may be problematic. Similar challenges may also be encountered in pathological states which may affect the relationship between the pool of growing and resting ovarian follicles. Given that previous histological investigations have suggested that a larger percentage of follicles are in the growing stages in older as compared with younger reproductive-aged women, some changes in these relationships should also be anticipated to naturally occur at the extremes of the reproductive lifespan. ${ }^{101}$

\section{Conclusions}

Predicting the age of the final menstrual period for the individual woman remains an important goal for clinicians and patients alike. In addition to risk assessment, the prediction of the age of menopause may well predict the age of subfertility and the end of natural fertility. Ideally the prediction of the reproductive lifespan would occur at a younger age, allowing for effective interventions to be undertaken. Recent histological and longitudinal investigations have demonstrated a significant relationship between biomarkers of ovarian reserve and the true ovarian reserve, as assessed by the ovarian PF count and the final menstrual period, respectively. Models predicting the age of menopause incorporating ovarian reserve markers, particularly serum AMH levels, have shown promise in the prediction of the reproductive lifespan. However, the relatively wide confidence intervals associated with the predictions generated by these models, particularly at the extremes of the age of anticipated menopause, currently limit their clinical utility. Further limiting the application of these models is the lack of international standards for the AMH assay and its low sensitivity. In summary, predicting the reproductive lifespan remains a goal that we have not yet reached. Future longitudinal and histological investigations, including those aimed at identifying new biomarkers, are necessary to improve the accuracy of models of reproductive aging.

\section{Acknowledgments}

The author would like to thank Dr. Michael Soules and Dr. Nancy Klein for their critical review of the manuscript. Additionally, we would like to acknowledge the NIH and the Oklahoma Center for the Advancement of Science and Technology for funding much of the work presented in this review.

\section{References}

1 te Velde ER, Pearson PL. The variability of female reproductive ageing. Hum Reprod Update 2002;8(2):141-154

2 Leridon H. Demographic effects of the introduction of steroid contraception in developed countries. Hum Reprod Update 2006; 12(5):603-616

3 Matthews TJ, Hamilton BE. Delayed childbearing: more women are having their first child later in life. NCHS Data Brief 2009; 21(21):1-8

4 Soules MR; American Society for Reproductive Medicine. The story behind the American Society for Reproductive Medicine's prevention of infertility campaign. Fertil Steril 2003;80(2): 295-299

5 van Wayenburg CA, van der Schouw YT, van Noord PA, Peeters $\mathrm{PH}$. Age at menopause, body mass index, and the risk of colorectal cancer mortality in the Dutch Diagnostisch Onderzoek Mammacarcinoom (DOM) cohort. Epidemiology 2000; 11(3):304-308 
6 van Der Voort DJ, van Der Weijer PH, Barentsen R. Early menopause: increased fracture risk at older age. Osteoporos Int 2003;14(6):525-530

7 van der Schouw YT, van der GraafY, Steyerberg EW, Eijkemans JC, Banga JD. Age at menopause as a risk factor for cardiovascular mortality. Lancet 1996;347(9003):714-718

8 Mondul AM, Rodriguez C, Jacobs EJ, Calle EE. Age at natural menopause and cause-specific mortality. Am J Epidemiol 2005;162(11):1089-1097

9 Ossewaarde ME, Bots ML, Verbeek AL, et al. Age at menopause, cause-specific mortality and total life expectancy. Epidemiology 2005;16(4):556-562

10 Monninkhof EM, van der Schouw YT, Peeters PH. Early age at menopause and breast cancer: are leaner women more protected? A prospective analysis of the Dutch DOM cohort. Breast Cancer Res Treat 1999;55(3):285-291

11 Fries JF. Aging, natural death, and the compression of morbidity. N Engl J Med 1980;303(3):130-135

12 Treloar AE. Menstrual cyclicity and the pre-menopause. Maturitas 1981;3(3-4):249-264

13 Broekmans FJ, Soules MR, Fauser BC. Ovarian aging: mechanisms and clinical consequences. Endocr Rev 2009;30(5):465-493

14 Santoro N, Brown JR, Adel T, Skurnick JH. Characterization of reproductive hormonal dynamics in the perimenopause. J Clin Endocrinol Metab 1996;81(4):1495-1501

15 Lambalk CB, van Disseldorp J, de Koning CH, Broekmans FJ. Testing ovarian reserve to predict age at menopause. Maturitas 2009;63(4):280-291

16 Weiss G, Skurnick JH, Goldsmith LT, Santoro NF, Park SJ. Menopause and hypothalamic-pituitary sensitivity to estrogen. JAMA 2004;292(24):2991-2996

17 Gougeon A. Regulation of ovarian follicular development in primates: facts and hypotheses. Endocr Rev 1996;17(2):121-155

18 Gougeon A. The early stages of follicular growth. In: Trounson AO, Gosden RG, eds. Biology and Pathology of the Oocyte: Role in Fertility and Reproductive Medicine. Cambridge: Cambridge University Press; 2003:29-41

19 Battaglia DE, Goodwin P, Klein NA, Soules MR. Influence of maternal age on meiotic spindle assembly in oocytes from naturally cycling women. Hum Reprod 1996;11(10):2217-2222

20 Munné S, Chen S, Colls P, et al. Maternal age, morphology, development and chromosome abnormalities in over 6000 cleavage-stage embryos. Reprod Biomed Online 2007;14(5): 628-634

21 Block E. Quantitative morphological investigations of the follicular system in women; variations at different ages. Acta Anat (Basel) 1952;14(1-2, Suppl 16):108-123

22 Gougeon A, Chainy GB. Morphometric studies of small follicles in ovaries of women at different ages. J Reprod Fertil 1987;81(2): 433-442

23 Faddy MJ, Gosden RG, Gougeon A, Richardson SJ, Nelson JF. Accelerated disappearance of ovarian follicles in mid-life: implications for forecasting menopause. Hum Reprod 1992;7(10): 1342-1346

24 Hansen KR, Knowlton NS, Thyer AC, Charleston JS, Soules MR, Klein NA. A new model of reproductive aging: the decline in ovarian non-growing follicle number from birth to menopause. Hum Reprod 2008;23(3):699-708

25 Gundersen HJ, Bagger P, Bendtsen TF, et al. The new stereological tools: disector, fractionator, nucleator and point sampled intercepts and their use in pathological research and diagnosis. APMIS 1988;96(10):857-881

26 West MJ. New stereological methods for counting neurons. Neurobiol Aging 1993;14(4):275-285

27 Wallace WHB, Kelsey TW. Human ovarian reserve from conception to the menopause. PLoS ONE 2010;5(1):e8772

28 Scott RT, Toner JP, Muasher SJ, Oehninger S, Robinson S, Rosenwaks Z. Follicle-stimulating hormone levels on cycle day 3 are predictive of in vitro fertilization outcome. Fertil Steril 1989; 51(4):651-654

29 Toner JP, Philput CB, Jones GS, Muasher SJ. Basal follicle-stimulating hormone level is a better predictor of in vitro fertilization performance than age. Fertil Steril 1991;55(4):784-791

30 Smotrich DB, Widra EA, Gindoff PR, Levy MJ, Hall JL, Stillman RJ. Prognostic value of day 3 estradiol on in vitro fertilization outcome. Fertil Steril 1995;64(6):1136-1140

31 Frattarelli JL, Bergh PA, Drews MR, Sharara FI, Scott RT. Evaluation of basal estradiol levels in assisted reproductive technology cycles. Fertil Steril 2000;74(3):518-524

32 Seifer DB, Lambert-Messerlian G, Hogan JW, Gardiner AC, Blazar AS, Berk CA. Day 3 serum inhibin-B is predictive of assisted reproductive technologies outcome. Fertil Steril 1997;67(1): $110-114$

33 Hall JE, Welt CK, Cramer DW. Inhibin A and inhibin B reflect ovarian function in assisted reproduction but are less useful at predicting outcome. Hum Reprod 1999;14(2):409-415

34 Tomas C, Nuojua-Huttunen S, Martikainen H. Pretreatment transvaginal ultrasound examination predicts ovarian responsiveness to gonadotrophins in in-vitro fertilization. Hum Reprod 1997; 12(2):220-223

35 Chang M-Y, Chiang C-H, Hsieh T-T, Soong Y-K, Hsu K-H. Use of the antral follicle count to predict the outcome of assisted reproductive technologies. Fertil Steril 1998;69(3):505-510

36 Frattarelli JL, Lauria-Costab DF, Miller BT, Bergh PA, Scott RT. Basal antral follicle number and mean ovarian diameter predict cycle cancellation and ovarian responsiveness in assisted reproductive technology cycles. Fertil Steril 2000;74(3):512-517

37 Bancsi LFJMM, Broekmans FJM, Eijkemans MJC, de Jong FH, Habbema JDF, te Velde ER. Predictors of poor ovarian response in in vitro fertilization: a prospective study comparing basal markers of ovarian reserve. Fertil Steril 2002; $77(2): 328-336$

38 Hansen KR, Morris JL, Thyer AC, Soules MR. Reproductive aging and variability in the ovarian antral follicle count: application in the clinical setting. Fertil Steril 2003;80(3):577-583

39 van Rooij IAJ, Broekmans FJ, te Velde ER, et al. Serum antiMüllerian hormone levels: a novel measure of ovarian reserve. Hum Reprod 2002;17(12):3065-3071

40 Seifer DB, MacLaughlin DT, Christian BP, Feng B, Shelden RM. Early follicular serum müllerian-inhibiting substance levels are associated with ovarian response during assisted reproductive technology cycles. Fertil Steril 2002;77(3):468-471

41 Broer SL, Mol BW, Hendriks D, Broekmans FJ. The role of antimullerian hormone in prediction of outcome after IVF: comparison with the antral follicle count. Fertil Steril 2009;91(3): 705-714

42 Loumaye E, Billion JM, Mine JM, Psalti I, Pensis M, Thomas K. Prediction of individual response to controlled ovarian hyperstimulation by means of a clomiphene citrate challenge test. Fertil Steril 1990;53(2):295-301

43 Tanbo T, Dale PO, Lunde O, Norman N, Abyholm T. Prediction of response to controlled ovarian hyperstimulation: a comparison of basal and clomiphene citrate-stimulated follicle-stimulating hormone levels. Fertil Steril 1992;57(4):819-824

44 Padilla SL, Bayati J, Garcia JE. Prognostic value of the early serum estradiol response to leuprolide acetate in in vitro fertilization. Fertil Steril 1990;53(2):288-294

45 Winslow KL, Toner JP, Brzyski RG, Oehninger SC, Acosta AA, Muasher SJ. The gonadotropin-releasing hormone agonist stimulation test-a sensitive predictor of performance in the flare-up in vitro fertilization cycle. Fertil Steril 1991;56(4):711-717

46 Broekmans FJ, Kwee J, Hendriks DJ, Mol BW, Lambalk CB. A systematic review of tests predicting ovarian reserve and IVF outcome. Hum Reprod Update 2006;12(6):685-718

47 Verhagen TE, Hendriks DJ, Bancsi LF, Mol BW, Broekmans FJ. The accuracy of multivariate models predicting ovarian reserve and 
pregnancy after in vitro fertilization: a meta-analysis. Hum Reprod Update 2008;14(2):95-100

48 Sun W, Stegmann BJ, Henne M, Catherino WH, Segars JH. A new approach to ovarian reserve testing. Fertil Steril 2008;90(6): 2196-2202

49 Scott RT Jr, Elkind-Hirsch KE, Styne-Gross A, Miller KA, Frattarelli $\mathrm{JL}$. The predictive value for in vitro fertility delivery rates is greatly impacted by the method used to select the threshold between normal and elevated basal follicle-stimulating hormone. Fertil Steril 2008;89(4):868-878

50 Hansen KR, Hodnett GM, Knowlton N, Craig LB. Correlation of ovarian reserve tests with histologically determined primordial follicle number. Fertil Steril 2011;95(1):170-175

51 Sherman BM, West JH, Korenman SG. The menopausal transition: analysis of LH, FSH, estradiol, and progesterone concentrations during menstrual cycles of older women. J Clin Endocrinol Metab 1976;42(4):629-636

52 Reyes FI, Winter JS, Faiman C. Pituitary-ovarian relationships preceding the menopause. I. A cross-sectional study of serum follicle-stimulating hormone, luteinizing hormone, prolactin, estradiol, and progesterone levels. Am J Obstet Gynecol 1977; 129(5):557-564

53 Klein NA, Houmard BS, Hansen KR, et al. Age-related analysis of inhibin $A$, inhibin $B$, and activin a relative to the intercycle monotropic follicle-stimulating hormone rise in normal ovulatory women. J Clin Endocrinol Metab 2004;89(6):2977-2981

54 Randolph JF Jr, Crawford S, Dennerstein L, et al. The value of follicle-stimulating hormone concentration and clinical findings as markers of the late menopausal transition. J Clin Endocrinol Metab 2006;91(8):3034-3040

55 Harlow SD, Gass M, Hall JE, et al; STRAW+10 Collaborative Group. Executive summary of the Stages of Reproductive Aging Workshop +10: addressing the unfinished agenda of staging reproductive aging. Climacteric 2012;15(2):105-114

56 Harlow SD, Gass M, Hall JE, et al; STRAW + 10 Collaborative Group. Executive summary of the Stages of Reproductive Aging Workshop +10 : addressing the unfinished agenda of staging reproductive aging. Fertil Steril 2012;97(4):843-851

57 Harlow SD, Gass M, Hall JE, et al; STRAW + 10 Collaborative Group. Executive summary of the Stages of Reproductive Aging Workshop +10 : addressing the unfinished agenda of staging reproductive aging. J Clin Endocrinol Metab 2012;97(4): 1159-1168

58 Harlow SD, Gass M, Hall JE, et al; STRAW 10 Collaborative Group. Executive summary of the Stages of Reproductive Aging Workshop +10 : addressing the unfinished agenda of staging reproductive aging. Menopause 2012;19(4):387-395

59 Sowers MR, Eyvazzadeh AD, McConnell D, et al. Anti-mullerian hormone and inhibin $B$ in the definition of ovarian aging and the menopause transition. J Clin Endocrinol Metab 2008;93(9): 3478-3483

60 Welt C, Sidis Y, Keutmann H, Schneyer A. Activins, inhibins, and follistatins: from endocrinology to signaling. A paradigm for the new millennium. Exp Biol Med (Maywood) 2002;227(9): 724-752

61 Vale W, Rivier C, Hsueh A, et al. Chemical and biological characterization of the inhibin family of protein hormones. Recent Prog Horm Res 1988;44:1-34

62 Weiss J, Crowley WFJ Jr, Halvorson LM, Jameson JL. Perifusion of rat pituitary cells with gonadotropin-releasing hormone, activin, and inhibin reveals distinct effects on gonadotropin gene expression and secretion. Endocrinology 1993;132(6):2307-2311

63 Groome NP, Illingworth PJ, O'Brien M, et al. Measurement of dimeric inhibin B throughout the human menstrual cycle. J Clin Endocrinol Metab 1996;81(4):1401-1405

64 Corson SL, Gutmann J, Batzer FR, Wallace H, Klein N, Soules MR. Inhibin-B as a test of ovarian reserve for infertile women. Hum Reprod 1999;14(11):2818-2821
65 Creus M, Peñarrubia J, Fábregues F, et al. Day 3 serum inhibin B and FSH and age as predictors of assisted reproduction treatment outcome. Hum Reprod 2000;15(11):2341-2346

66 Gracia CR, Sammel MD, Freeman EW, et al. Defining menopause status: creation of a new definition to identify the early changes of the menopausal transition. Menopause 2005;12(2):128-135

67 Burger HG, Hale GE, Robertson DM, Dennerstein L. A review of hormonal changes during the menopausal transition: focus on findings from the Melbourne Women's Midlife Health Project. Hum Reprod Update 2007;13(6):559-565

68 Scheffer GJ, Broekmans FJ, Dorland M, Habbema JD, Looman CW, te Velde ER. Antral follicle counts by transvaginal ultrasonography are related to age in women with proven natural fertility. Fertil Steril 1999;72(5):845-851

69 Rosen MP, Sternfeld B, Schuh-Huerta SM, Reijo Pera RA, McCulloch CE, Cedars MI. Antral follicle count: absence of significant midlife decline. Fertil Steril 2010;94(6):2182-2185

70 Yang YS, Hur MH, Kim SY, Young K. Correlation between sonographic and endocrine markers of ovarian aging as predictors for late menopausal transition. Menopause 2011;18(2):138-145

71 Hansen KR, Craig LB, Zavy MT, Klein NA, Soules MR. Ovarian primordial and nongrowing follicle counts according to the Stages of Reproductive Aging Workshop (STRAW) staging system. Menopause 2012;19(2):164-171

72 Hehenkamp WJ, Looman CW, Themmen AP, de Jong FH, Te Velde ER, Broekmans FJ. Anti-Müllerian hormone levels in the spontaneous menstrual cycle do not show substantial fluctuation. J Clin Endocrinol Metab 2006;91(10):4057-4063

73 Weenen C, Laven JS, Von Bergh AR, et al. Anti-Müllerian hormone expression pattern in the human ovary: potential implications for initial and cyclic follicle recruitment. Mol Hum Reprod 2004;10 (2):77-83

74 Fanchin R, Schonäuer LM, Righini C, Guibourdenche J, Frydman R, Taieb J. Serum anti-Müllerian hormone is more strongly related to ovarian follicular status than serum inhibin B, estradiol, FSH and LH on day 3. Hum Reprod 2003;18(2):323-327

75 Hale GE, Zhao X, Hughes CL, Burger HG, Robertson DM, Fraser IS. Endocrine features of menstrual cycles in middle and late reproductive age and the menopausal transition classified according to the Staging of Reproductive Aging Workshop (STRAW) staging system. J Clin Endocrinol Metab 2007;92(8):3060-3067

76 van Rooij IA, Broekmans FJ, Scheffer GJ, et al. Serum antimullerian hormone levels best reflect the reproductive decline with age in normal women with proven fertility: a longitudinal study. Fertil Steril 2005;83(4):979-987

77 Seifer DB, Baker VL, Leader B. Age-specific serum anti-Müllerian hormone values for 17,120 women presenting to fertility centers within the United States. Fertil Steril 2011;95(2):747-750

78 van Disseldorp J, Faddy MJ, Themmen APN, et al. Relationship of serum antimüllerian hormone concentration to age at menopause. J Clin Endocrinol Metab 2008;93(6):2129-2134

79 Kelsey TW, Wright P, Nelson SM, Anderson RA, Wallace WH. A validated model of serum anti-müllerian hormone from conception to menopause. PLoS ONE 2011;6(7):e22024

80 de Vet A, Laven JS, de Jong FH, Themmen AP, Fauser BC. Antimüllerian hormone serum levels: a putative marker for ovarian aging. Fertil Steril 2002;77(2):357-362

81 Tran ND, Cedars MI, Rosen MP. The role of anti-müllerian hormone (AMH) in assessing ovarian reserve. J Clin Endocrinol Metab 2011;96(12):3609-3614

82 de Boer EJ, den Tonkelaar I, te Velde ER, Burger CW, van Leeuwen FE; OMEGA-project group. Increased risk of early menopausal transition and natural menopause after poor response at first IVF treatment. Hum Reprod 2003;18(7):1544-1552

83 Lawson R, El-Toukhy T, Kassab A, et al. Poor response to ovulation induction is a stronger predictor of early menopause than elevated basal FSH: a life table analysis. Hum Reprod 2003;18 (3):527-533 
84 Nikolaou D, Lavery S, Turner C, Margara R, Trew G. Is there a link between an extremely poor response to ovarian hyperstimulation and early ovarian failure? Hum Reprod 2002;17(4): 1106-1111

85 Gülekli B, Bulbul Y, Onvural A, et al. Accuracy of ovarian reserve tests. Hum Reprod 1999;14(11):2822-2826

86 Treloar AE, Boynton RE, Behn BG, Brown BW. Variation of the human menstrual cycle through reproductive life. Int J Fertil 1967;12(1, Pt 2):77-126

87 Taffe JR, Dennerstein L. Menstrual patterns leading to the final menstrual period. Menopause 2002;9(1):32-40

88 Mitchell ES, Woods NF, Mariella A. Three stages of the menopausal transition from the Seattle Midlife Women's Health Study: toward a more precise definition. Menopause 2000;7(5): 334-349

89 Harlow SD, Mitchell ES, Crawford S, Nan B, Little R, Taffe J; ReSTAGE Collaboration. The ReSTAGE Collaboration: defining optimal bleeding criteria for onset of early menopausal transition. Fertil Steril 2008;89(1):129-140

90 Taffe JR, Cain KC, Mitchell ES, Woods NF, Crawford SL, Harlow SD. "Persistence" improves the 60-day amenorrhea marker of entry to late-stage menopausal transition for women aged 40 to 44 years. Menopause 2010;17(1):191-193

91 Klein NA, Harper AJ, Houmard BS, Sluss PM, Soules MR. Is the short follicular phase in older women secondary to advanced or accelerated dominant follicle development? J Clin Endocrinol Metab 2002;87(12):5746-5750

92 Soules MR, Sherman S, Parrott E, et al. Executive Summary: Stages of reproductive aging workshop (STRAW). Fertil Steril 2001; 76(5):874-878
93 Lass A. Assessment of ovarian reserve: is there still a role for ovarian biopsy in the light of new data? Hum Reprod 2004;19(3): 467-469

94 Broekmans FJ, Faddy MJ, Scheffer G, te Velde ER. Antral follicle counts are related to age at natural fertility loss and age at menopause. Menopause 2004;11(6, Pt 1):607-614

95 van Rooij IA, Tonkelaar Id, Broekmans FJ, et al. Anti-müllerian hormone is a promising predictor for the occurrence of the menopausal transition. Menopause 2004;11(6, Pt 1):601-606

96 Broer SL, Eijkemans MJC, Scheffer GJ, et al. Anti-mullerian hormone predicts menopause: a long-term follow-up study in normoovulatory women. J Clin Endocrinol Metab 2011;96(8): 2532-2539

97 Tehrani FR, Shakeri N, Solaymani-Dodaran M, Azizi F. Predicting age at menopause from serum antimüllerian hormone concentration. Menopause 2011;18(7):766-770

98 Tehrani FR, Solaymani-Dodaran M, Tohidi M, Gohari MR, Azizi F. Modeling age at menopause using serum concentration of antimullerian hormone. J Clin Endocrinol Metab 2013;98(2):729-735

99 Freeman EW, Sammel MD, Lin H, Gracia CR. Anti-mullerian hormone as a predictor of time to menopause in late reproductive age women. J Clin Endocrinol Metab 2012;97(5):1673-1680

100 Freeman EW, Sammel MD, Lin H, Boorman DW, Gracia CR. Contribution of the rate of change of antimüllerian hormone in estimating time to menopause for late reproductive-age women. Fertil Steril 2012;98(5):1254-1259, e1-e2

101 Gougeon A, Ecochard R, Thalabard JC. Age-related changes of the population of human ovarian follicles: increase in the disappearance rate of non-growing and early-growing follicles in aging women. Biol Reprod 1994;50(3):653-663 